\title{
EFFECTS OF SOURCE STRUCTURE ON ASTROMETRY AND GEODESY
}

\author{
James S. Ulvestad \\ Jet Propulsion Laboratory \\ California Institute of Technology \\ Pasadena, California 91109
}

\begin{abstract}
VLBI hybrid maps of a number of radio sources have been used to make calculations of the effects of source structure on group delay and phase delay for baselines of different lengths. The effects on the two types of delay are of comparable magnitude and have a slightly stronger than linear dependence on baseline length. For sources selected to have only modest structure, the r.m.s. delay error caused by structure at $8.4 \mathrm{GHz}$ is on the order of $\mathbf{3 0}$ to 50 picoseconds for intercontinental baselines.
\end{abstract}

A limit to the accuracy of astrometric and geodetic observations of compact radio sources is provided by the non-pointlike structures of some such sources. The nature of the source structure "error" is somewhat different in group and phase delay. At $(u, v)$ points where the fringe visibility is low, the group delay caused by the source structure may be very large, while it is relatively small at high visibility points. The phase delay, on the other hand, will typically undergo large changes at low visibility points. In a region of high fringe visibility, the phase delay caused by structure may be fairly constant, but may be quite different from that in another high-visibility region in the $(u, v)$ plane.

Source structure effects at $8.4 \mathrm{GHz}$ ( $\mathrm{X}$ band) have been calculated using 5-GHz maps of 26 sources from the first epoch of the Caltech VLBI survey (Pearson and Readhead 1984; Pearson, Readhead, and Barthel, this volume). These calculations have been made by considering each source to be a collection of point sources, as given by the model components in a hybrid map.

Calculations of structure effects on delay have been made every three minutes along the $(u, v)$ tracks for each source on three different baselines. The $(u, v)$ tracks for the real (northerly) declinations of the sources have been used. The three baselines used in the computations are Goldstone to Madrid $(8390 \mathrm{~km})$, Fort Davis to Haystack $(3140 \mathrm{~km})$, and Green Bank to Haystack $(840 \mathrm{~km})$. For the purposes of the calculations, the $8.4-\mathrm{GHz}$ structures have been assumed to be identical to the 5.0-GHz structures. Therefore the calculations for individual sources are not correct in detail, but the computations are used only for statistical analysis and not for detailed corrections for individual source structures. Since the structure that would actually be seen at $8.4 \mathrm{GHz}$ would be smaller and more core-dominated than at $5 \mathrm{GHz}$, the structure effects are probably overestimated by a factor of 1.5 to 2 . 
Although the two shorter baselines were actually used in making the hybrid maps, the Goldstone-Madrid baseline was not. However, almost all the maps used had data from the baseline between Owens Valley and Bonn, which is not very different from the Goldstone-Madrid baseline. The major source of uncertainty is the interpolation to unsampled parts of the $(u, v)$ plane because the frequency used for calculations was not the same as that used in making the maps.

For the full set of 26 sources used, the r.m.s. errors due to unmodeled source structure are misleadingly large because of the inclusion of sources that have so much structure that they generally would not be present in astrometric or geodetic VLBI catalogs. Therefore, a subset of 16 "good" astrometric sources has been constructed, from which 3C 84 and all sources classified as compact doubles or steep-spectrum cores by Pearson and Readhead (1984) have been eliminated.

TABLE 1. Calculated RMS Structure Delays for 16 "Good” Sources

\begin{tabular}{lccc}
\hline Baseline & Length & Group Delay & Phase Delay \\
& & & \\
Goldstone-Madrid & $8390 \mathrm{~km}$ & $83 \mathrm{psec}$ & $56 \mathrm{psec}$ \\
Haystack-Fort Davis & $3140 \mathrm{~km}$ & $28 \mathrm{psec}$ & $19 \mathrm{psec}$ \\
Haystack-Green Bank & $840 \mathrm{~km}$ & $5 \mathrm{psec}$ & $3 \mathrm{psec}$ \\
\hline
\end{tabular}

Table 1 shows the r.m.s. delays caused by structure of the 16 sources on the three baselines studied. The group delay is calculated relative to the flux density centroid of each source, while the phase delay is calculated relative to the mean value for each source. The r.m.s. group delays range from 5 picoseconds on the shortest baseline to 83 picoseconds on the longest baseline, while the r.m.s. phase delays are about $30-40 \%$ lower. As mentioned above, these calculated values are probably overestimates by factors of 1.5 to 2 . The higher r.m.s. for the group delays is probably caused by the extended tail of the structure delay distribution, which in turn is related to the behavior of the group delay near visibility minima. Power law fits of delay vs. baseline length give exponents of 1.19 for the group delay and 1.28 for the phase delay over the factor of 10 in baseline length studied here. The approximate scaling with baseline length indicates that uncorrected source structure will cause astrometric errors at the level of a several tenths of a milliarcsecond for the range of baselines considered. Preliminary indications are that power laws with similar indices continue down to baselines of several hundred kilometers.

I thank Tim Pearson for supplying the hybrid maps used in this work.

\section{REFERENCES}

1. Pearson, T. J., and Readhead, A. C. S.: 1984, IAU Symp. No. 110, p. 15.

2. Pearson, T. J., Readhead, A. C. S., and Barthel, P. D.: 1987, IAU Symp. No. 129, this volume. 